\title{
Design and Test of a New Type Amplifier-based Sensing Device for High Temperature Deformation Monitoring
}

\author{
Jiu-Hong Jia ${ }^{1, a}$, Shu-Jun Duan ${ }^{2, b}$, Xiao-Yin $\mathrm{Hu}^{3, \mathrm{c}}$, Shan-Tung $\mathrm{Tu}^{4, \mathrm{~d}}$ \\ ${ }^{1,2,3,4}$ Key Laboratory of Pressure Systems and Safety, Ministry of Education, East China University \\ of Science and Technology, Shanghai, 200237, China \\ ajhjia@ecust.com, bdsj89623@sina.com, caya277@qq.com, dsttu@ecust.edu.cn
}

\begin{abstract}
Keywords: on-line strain monitoring; small deformation; sensing device; displacement amplifier. Abstract: In order to solve the problem in monitoring high temperature strain coming from harsh working environment and extremely small deformation of components, a flexure-hinged displacement amplifier is proposed to be integrated in a sensing device in order to design a more sensitive sensor to accurately online monitor small deformation of high temperature components. The structure and the work principle of the sensing device are introduced. A prototype of the sensing device is fabricated. Then the tests to verify the reliability and amplification ability are carried out at laboratory. Results demonstrate that the designed sensing device has satisfactory working performance.
\end{abstract}

\section{Introduction}

High temperature components are widely applied in aviation, power generation, petroleum and chemical industries. To obtain the relationship between deformation and residual life of components in many cases, the strain measurement has been the most straightforward and reliable method for life monitoring [1-3].

Main challenges in monitoring high temperature strain come from harsh working environment and extremely small deformation of components. Commercial high temperature strain gages can hardly work under a temperature over $500^{\circ} \mathrm{C}$ for long time monitoring [4]. Although many kinds of direct sensors have been developed, their applications are limited to the temperature below $500^{\circ} \mathrm{C}$ and short operation periods [5-8]. As a better alternative solution, extension-based sensing devices are designed and verified $[9,10]$. Test results show that the designed devices can work at elevated temperature. However, the accuracy and resolution of the device are not sufficient enough for measuring very small creep strain of in-service high temperature components, which is normally less than $0.8 \times 10-5 \% / \mathrm{h}[10]$. Therefore, in this paper, a flexure-hinged mechanical amplifier, the characteristics of which have been studied in [11], is proposed to be integrated with a sensing device. The structure and working principle of the sensing device is depicted in Section 2. High temperature experimental verification is depicted in Section 3. Conclusions are given in Section 4.

\section{Structure and Working Principle of the New Sensing device}

A schematic map of the sensing device mounted on a specimen is shown in Fig. 1. The sensing device is exclusively self-supporting, which can easily measure the deformation of any part of a component. It is composed of two transmission arms, two connecting pieces, a displacement amplifier and a sensor, as shown in Fig. 1.

The transmission arms are mounted on the surface of the specimen by welding or banding. The included angle between connecting piece and transmission arm can be adjusted in the scope of [0, $90^{\circ}$ ] for different measurements. Owing to the possibility of angular adjustment of connecting pieces, the sensing device is applicable not only to the test pieces with flat surfaces, but also to curve surface specimens such as pipes. The displacement amplifier is assembled between two connecting pieces. Because all parts of the transmission mechanism are connected rigidly, the distance between the point $C$ and $D$ will change synchronously when the test specimen is loaded in $\mathrm{AB}$ direction. The distance change between $\mathrm{C}$ and $\mathrm{D}$ will give rise to a vertical output displacement 
of the amplifier owing to the special work performance [11]. And the output value can be measured by the sensor in time.

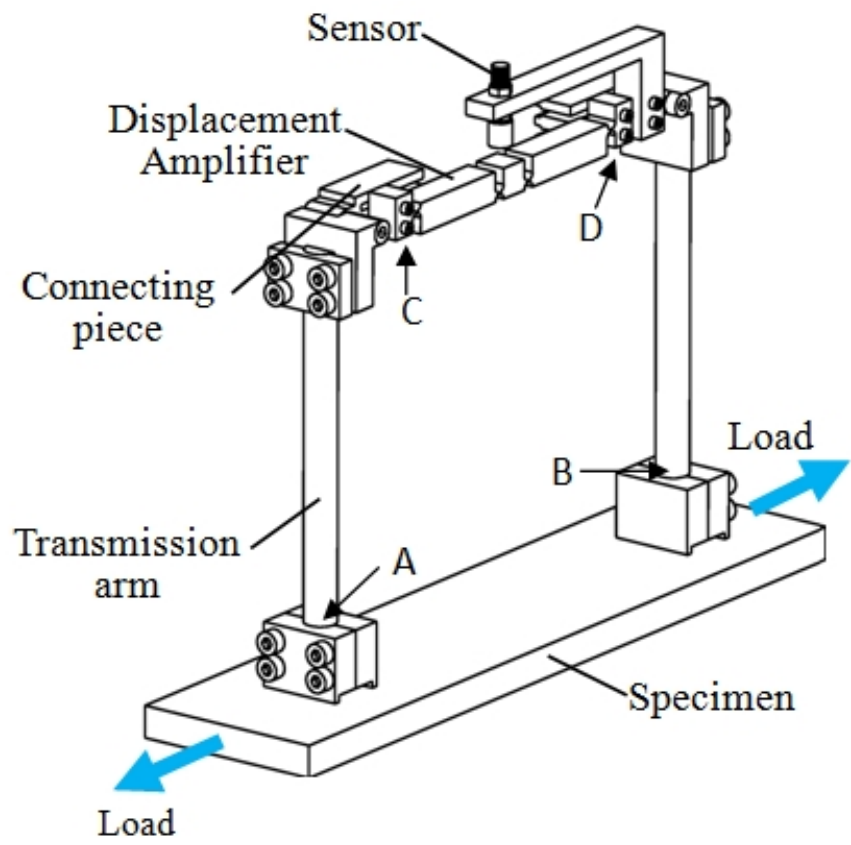

Fig. 1 Structure of the new sensing device

The sensor applied in this sensing device can be an eddy-current transducer, named AEC-PU20. Because the eddy current sensor for static strain measurement requires a target, whose size is twice as large as the sensor diameter, a $2 \mathrm{~mm}$ thick cylinder that has a diameter of $40 \mathrm{~mm}$ is mounted on the sensing device.

The space between the displacement amplifier and the specimen needs a minor removal of the thermal insulation materials that is used to decrease the heat loss of the high temperature specimen. On this condition, the connecting pieces, the displacement amplifier and the sensor are working outside the insulation layer.

\section{High Temperature Experimental Verification}

To verify the performance of the designed sensing device, a prototype is produced. And an experiment system is set up, shown in Fig. 2, which is nearly the same with the test system in References [9]. The device in the red dashed line box is the designed sensing device, which is mounted to the specimen by fixing blocks. The specimen is installed in an insulation box. The heater inside the insulation box can heat the specimen to $540^{\circ} \mathrm{C}$.

The sensing device is installed on both ends of a round bar test piece by fixing blocks. When pulling machine loading, the specimen is stretched. The change of distance between fixing blocks is the input displacement of the sensing device. In order to appraise the measurement accuracy of the sensing device, a calibration sensor (ZKL-X-15) is used, and a metal rod is added to suit the range of the sensing device. The calibration sensor and the metal rod are located on one side of the specimen. When the specimen is loaded, the calibration sensor and the metal rod will follow the change of the position blocks rigidly. Therefore, the measured value of the calibration sensor can serves as the real change value of the test piece. The sensor applied in this sensing device is an eddy-current transducer, named AEC-PU20. The performance of the sensors applied in the experiment is shown in table 1. 


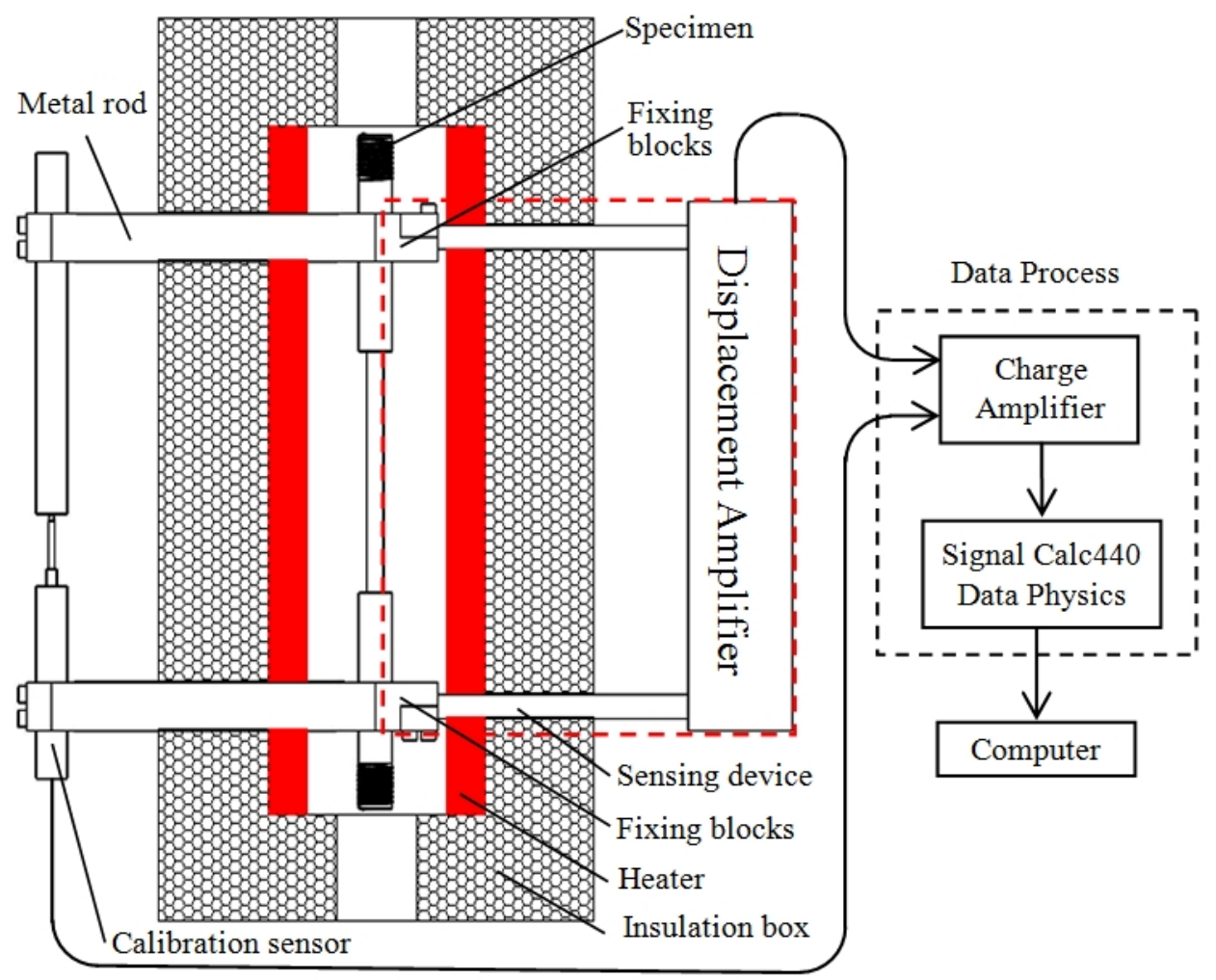

Fig.2 Schematic diagram of the test setup

Table 1 Performance of the sensors

\begin{tabular}{c|c|c|c|l}
\hline Model & Rated Capacity & Temperature Range & Resolution & Nonlinearity \\
\hline AEC-PU20 & $8 \mathrm{~mm}$ & $-10 \sim 60^{\circ} \mathrm{C}$ & $3 \mu \mathrm{m}$ & With $\pm 1 \%$ \\
\hline ZKL-X-15 & $10 \mathrm{~mm}$ & $-10 \sim 80^{\circ} \mathrm{C}$ & $1 \mu \mathrm{m}$ & With $\pm 1 \%$ \\
\hline
\end{tabular}



Fig. 3 Experimental results of the sensing device.

During the test, the specimen is loaded freely by a tensile machine. In this test, a displacement about $100 \mu \mathrm{m}$ was added for each step until the displacement load reaches $1000 \mu \mathrm{m}$. When the data on the computer keeps stable, the results on the sensing device and calibration sensor were recorded. To get reliable measurement results, the whole test procedure was carried out three times. Results for all these tests are drawn and compared in Fig. 3. 
From Fig. 3, it can be seen that three sets of experimental results match well and the results show that the sensing device has a linear displacement output and a constant amplification ratio, which is 3.75. The amplification ratio calculated basing on the test is approximately equal to the theoretical result.

\section{Conclusions}

In this paper, a sensing device integrating the mechanical displacement amplifier for high temperature strain monitoring is designed. Structure and working principle of the new sensing device is introduced, and its applicability is verified through tests. The output displacement of the sensing device is linear and repeatable. And deformation of the test piece can be accurately and robustly magnified 3.75 times by the amplifier-based sensing device. The experimental results verify that the sensing device can work stably and linearly. Therefore, the designed amplifier-based sensing device is reliable.

\section{Acknowledge}

The authors are grateful for the support of Pujiang Shanghai Talent Program (No. 15PJD010).

\section{References}

[1] B. Cane: Remaining creep life estimation by strain assessment on plant, International Journal of Pressure Vessels and Piping, 10(1982) 11-30.

[2] L. Chen, J.L. Jiang, Z.C. Fan, X.D. Chen, T.C. Yang: A new model for life prediction of fatigue-creep interaction, Int J Fatigue, 29(2007) 615-9.

[3] M.V. Borodii, M.P. Adamchuk: Life assessment for metallic materials with the use of the strain criterion for low cycle fatigue, Int J Fatigue, 31(2009) 1579-87.

[4] N.A. Riza, M. Sheikh, F. Perez: Hybrid wireless-wired optical sensor for extreme temperature measurement in next generation energy efficient gas turbines, J Eng Gas Turb Power, 132(2010).

[5] X.Y. Hu, J.H. Jia, N. Wang, X.M. Xia, Z.D. Wang, S.T. Tu: Design and test of an sensing device for strain monitoring of high temperature pipelines, Journal of Pressure Vessel Technology, 134(2012) 044501.

[6] S.T. Tu, J.M. Gong, X. Ling, X.Y. He: Measurement of local creep deformation in cross-weld specimen by optical fiber marking and remote monitoring, J Press Vess-T Asme, 124(2002) 54-8.

[7] A. Morris, J. Dear, M. Kourmpetis: High temperature steam pipelines- development of the ARCMAC creep monitoring system, Strain, 42(2006) 181-5.

[8] A. Morris, M. Kourmpetis, I.D. Dear, M. Sjodahl, J.P. Dear: Optical strain monitoring techniques for life assessment of components in power generation plants, P I Mech Eng a-J Pow, 221(2007) 1141-52.

[9] J.H. Jia, X.Y. Hu, Z.L. An, F.Z. Xuan, S.T. Tu: Design and verification of a sensing device for deformation measurement of high temperature pipes, J Press Vess-T Asme, 133(2011).

[10] J.H. Jia, X.Y. Hu, N. Wang, S.T. Tu: Test verification of an sensing device for deformation measurement of high temperature straight pipes, Measurement, 45(2012) 1933-6.

[11] H.W. Ma, S. M. Yao, L.Q. Wang, Z. Zhong: Analysis of the displacement amplification ratio of bridge-type flexure hinge. Sensors and Actuators A, 132 (2006) 730-736. 